\title{
Probabilistic analysis of Online Bin Coloring algorithms via stochastic comparison
}

Citation for published version (APA):

Hiller, B., \& Vredeveld, T. (2008). Probabilistic analysis of Online Bin Coloring algorithms via stochastic comparison. METEOR, Maastricht University School of Business and Economics. METEOR Research Memorandum No. 006 https://doi.org/10.26481/umamet.2008006

Document status and date:

Published: 01/01/2008

DOI:

10.26481/umamet.2008006

Document Version:

Publisher's PDF, also known as Version of record

\section{Please check the document version of this publication:}

- A submitted manuscript is the version of the article upon submission and before peer-review. There can be important differences between the submitted version and the official published version of record.

People interested in the research are advised to contact the author for the final version of the publication, or visit the DOI to the publisher's website.

- The final author version and the galley proof are versions of the publication after peer review.

- The final published version features the final layout of the paper including the volume, issue and page numbers.

Link to publication

\footnotetext{
General rights rights.

- You may freely distribute the URL identifying the publication in the public portal. please follow below link for the End User Agreement:

www.umlib.nl/taverne-license

Take down policy

If you believe that this document breaches copyright please contact us at:

repository@maastrichtuniversity.nl

providing details and we will investigate your claim.
}

Copyright and moral rights for the publications made accessible in the public portal are retained by the authors and/or other copyright owners and it is a condition of accessing publications that users recognise and abide by the legal requirements associated with these

- Users may download and print one copy of any publication from the public portal for the purpose of private study or research.

- You may not further distribute the material or use it for any profit-making activity or commercial gain

If the publication is distributed under the terms of Article $25 \mathrm{fa}$ of the Dutch Copyright Act, indicated by the "Taverne" license above, 
Benjamin Hiller, Tjark Vredeveld

Probabilistic analysis of Online Bin Coloring algorithms via Stochastic Comparison

$\mathrm{RM} / 08 / 006$

JEL code: C61

\section{METEबrR}

Maastricht research school of Economics

of TEchnology and ORganizations

Universiteit Maastricht

Faculty of Economics and Business Administration P.O. Box 616

NL - 6200 MD Maastricht

phone : ++31433883830

fax : ++31433884873 


\title{
Probabilistic analysis of Online Bin Coloring algorithms via Stochastic Comparison
}

\author{
Benjamin Hiller* \\ Zuse Institute Berlin \\ Takustraße 7 \\ D-14195 Berlin \\ Germany \\ hiller@zib.de
}

\author{
Tjark Vredeveld \\ Maastricht University \\ Faculty of Economics and Business Administration \\ 6200 MD Maastricht \\ The Netherlands \\ t.vredeveld@ke.unimaas.nl
}

April 14, 2008

\begin{abstract}
This paper proposes a new method for probabilistic analysis of online algorithms that is based on the notion of stochastic dominance. We develop the method for the Online Bin Coloring problem introduced in [15]. Using methods for the stochastic comparison of Markov chains we establish the strong result that the performance of the online algorithm GREEDYFIT is stochastically dominated by the performance of the algorithm ONEBIN for any number of items processed. This result gives a more realistic picture than competitive analysis and explains the behavior observed in simulations.
\end{abstract}

\section{Introduction}

We propose a new method for probabilistic analysis of online algorithms by using the concept of stochastic dominance. The traditional approach for analyzing online algorithms is competitive analysis [27,5], which characterizes an online algorithm by its competitive ratio, i. e., the ratio of the objective value achieved by the online algorithm to the objective value of an optimal offline solution. Online algorithms are then compared by comparing their competitive ratios, i. e., a smaller competitive ratio is better for a minimization problem. One drawback of competitive analysis is that it often provides rather pessimistic results due to its worst-case character. This is partly overcome by more elaborate variants like averagecase competitive analysis [22] and smoothed competitive analysis [3, 21].

In our approach, we suggest to compare the performance of algorithms on random input sequences directly using stochastic dominance. A random variable $X$ is said to be stochastically dominated by a random variable $Y$, written $X \leq_{\text {st }} Y$, if

$$
\operatorname{Pr}[X \geq x] \leq \operatorname{Pr}[Y \geq x] \text { for all } x \in \mathbb{R} .
$$

Suppose we can describe the performance of two online algorithms $\mathcal{A}$ and $\mathcal{B}$ by random variables $\chi^{\mathcal{A}}$ and $\chi^{\mathcal{B}}$, respectively. We can then say that $\mathcal{A}$ is stochastically better than $\mathcal{B}$ (for a minimization problem), if $\chi^{\mathcal{A}} \leq_{\text {st }} \chi^{\mathcal{B}}$.

Stochastic comparison methods have been successfully applied in areas like queueing theory [25], finance, economics and in particular decision under risk [19]. In this paper we introduce these concepts to the study of online algorithms.

We use this approach to study algorithms for the Online Bin Coloring problem, which was introduced by Krumke et al. [15]. It has applications in commissioning [15], vehicle routing [7] and networking [16]; later in this paper we will describe an application to elevator control, too. The Bin Coloring problem is the following. The input consists of a sequence of unit-size items, each of which has one of $C$ colors. These items need to be packed sequentially into one of $m$ initially empty bins of capacity $B$. As soon as a bin is full, i.e., has exactly $B$ items, it is replaced by an empty one. The goal is to minimize the maximum number of different colors in one bin. We will refer to the number of different colors in a bin

\footnotetext{
*Supported by the DFG research group "Algorithms, Structure, Randomness" (Grant number GR 883/10-3, GR 883/104) and a DAAD dissertation grant.
} 
as its colorfulness. In the online version, the items arrive one by one and must be irrevocably assigned to a bin before the next item becomes known.

A natural algorithm for this problem is the algorithm GREEDYFit [15]. GREEDYFiT packs an item with an already present color in the bin with that color and otherwise chooses a bin which currently has the least number of different colors. Another simple algorithm, ONEBIN, packs all items in the same bin. Krumke et al. [15] analyzed these algorithms, showing the counterintuitive result that the trivial algorithm ONEBIN is better than the more sophisticated algorithm GREEDYFIT in terms of the competitive ratio. The authors mentioned that the most challenging issue is to analyze the algorithms from an average-case point of view to explain the clear dominance of GREEDYFiT over ONEBIN observed in simulations. Such an average-case analysis is implied by our results.

Our Results We propose a new probabilistic analysis of both deterministic and randomized online algorithms. As far as we know, this is the first use of stochastic dominance in the analysis of the quality of online and approximation algorithms.

Using this approach, we obtain strong results for the comparison of the GREEDyFiT and ONEBIN algorithms, which explain the superiority of GREEDYFit over OnEBIN observed in simulations. For our analysis, we assume that the color sequences are generated by choosing the color i.i.d. according to a color distribution $\gamma$. Note that in this model, all online algorithms eventually have to produce a bin with colorfulness $B$ if the number of colors is sufficiently high, say $C \geq 2 m B$. This implies that in this case, all online algorithms are asymptotically equally bad. Moreover, since eventually there will be a color subsequence of length $2 m B$ with all colors different, the asymptotic competitive ratio is 1 with probability 1 . Both issues indicate that asymptotic probabilistic analysis does not give meaningful results. We therefore show that GREEDYFIT is stochastically better than ONEBIN after $n$ items. To be more precise, let the random variables $\chi_{n}^{\mathrm{GF}}$ and $\chi_{n}^{\mathrm{OB}}$ denote the maximum colorfulness attained after processing $n$ items using GREEDYFit and ONEBIN, respectively. We show that $\chi_{n}^{\mathrm{GF}} \leq_{\text {st }} \chi_{n}^{\mathrm{OB}}$ for all $n$ and distributions $\gamma$. We also obtain a similar result if the objective is the average colorfulness instead of the maximum colorfulness. Both results are based on an analysis of Markov chains related to the algorithms.

We emphasize several implications of this result. Stochastic dominance implies not only that the expected value of GREEDYFiT is bounded by that of ONEBIN, but also that the expected competitive ratio of GREEDYFIT is not more than that of ONEBIN. The expected competitive ratio [22] is defined as the expectation of the ratio between online algorithm and offline optimum. By considering the uniform color distribution, our result can be interpreted as a counting result stating that there are more instances for which GREEDYFIT manages to achieve a low maximum colorfulness than for ONEBIN. If the Online Bin Coloring occurs as a subproblem and the overall performance depends in a non-decreasing way on the colorfulness achieved, than using GREEDYFit is better in expectation than using ONEBIN w. r. t. to overall performance.

Related work Various alternatives to standard competitive analysis have been proposed, almost all of them are based on the idea of weakening the offline adversary. This can be done by considering randomized online algorithms [5] or by allowing the online algorithms to use more resources [11]. More related to our approach are concepts like the diffuse adversary [14], average-case competitive analysis [22] and smoothed competitive analysis [3, 21], which are also based on random request sequences.

The Bin Coloring problem has been studied in [15, 7, 16]. It is shown in [7] and [16] that the offline version is NP-hard and that it cannot be approximated within a factor of $4 / 3$ unless $\mathrm{P}=\mathrm{NP}$. Lin et al. [16] provide an algorithm that finds a solution of cost OPT +1 in the case that there are exactly $m B$ items. For the online version, Krumke et al. [15] show that the competitive ratio of GREEDYFiT is at least $2 m$, the competitive ratio of ONEBIN is at most $2 m-1$, and that the competitive ratio of any randomized algorithm is $\Omega(m)$ even if it is allowed to use more than $m$ bins simultaneously. The Bin Coloring problem is also related to class-contrained knapsack problems [23, 24]. In those versions of the knapsack problem, each item is characterized by a size and a color and each knapsack has an additional limit on the number of different colors that it can hold.

Although Markov chains are a natural tool for the study of online algorithms, they have not been used much so far in this context. One prominent example is the paging problem, where Karlin et al. [12] studied request sequences generated by a Markov chain. However, this work uses Markov chains to model the instances and not for analyzing algorithms (although the theory of Markov decision processes is used to derive lower bounds for all online algorithms). Initiated by Coffman et al. [9], online bin packing algorithms have been analyzed by modelling their behavior by Markov chains [13, 1, 20]. General methods 
for the analysis of Markov chains have then been used to prove results for online algorithms. In contrast to our approach these results are only asymptotic and expectation-based. Other uses of Markov chains in the analysis of algorithms are in the field of approximate sampling [26]. Some of the techniques used there are similar to ours since they are based on the concept of coupling, which is very useful to compare probability distributions.

There are many applications of the rich theory of stochastic comparison and stochastic dominance, see e.g., [25, 19]. However, there are only few papers applying them to analyze or develop algorithms. The papers by Mitzenmacher [6, 18] employ these methods to analyze routing algorithms. Mitzenmacher states on his homepage that he thinks "stochastic dominance is under-utilized in computer science".

Structure of the paper Section 2 defines the problem variants and discusses an application to elevator control. Markov chain models for the online algorithms, basic notation and technical preliminaries are introduced in Section 3, including Theorem 1 which is the basis of our analysis. In Sections 4 and 5 we prove the result for minimizing the maximum and average colorfulness, respectively.

\section{Problem definition and an application to elevator control}

An instance of the Bin Coloring problem is described by the number of bins $m$, the bin capacity $B$ and the number of colors $C$. In the online probabilistic version there is also a probability distribution $\gamma$ over the colors given. Color sequences are generated by chosing each color according to $\gamma$ independently, i. e., the resulting color sequences are i.i.d.

As mentioned before, asymptotic analysis does not give meaningful results. However, the algorithms GreEdyFit and ONEBin differ in how long they manage to produce bins of low colorfulness. We therefore will analyze the transient instead of the asymptotic behavior of the algorithms. Let the random variables $\chi_{n}^{\mathrm{GF}}$ and $\chi_{n}^{\mathrm{OB}}$ denote the maximum colorfulness attained after processing $n$ items using GreedyFit and OneBin, respectively. We will show that GreEDyFit is stochastically better than ONEBIN after $n$ items, i.e., that $\chi_{n}^{\mathrm{GF}} \leq_{\text {st }} \chi_{n}^{\mathrm{OB}}$.

We will also show a similar result for another variant of the Online Bin Coloring problem, where the objective is to minimize the average colorfulness of the bins. Equivalently, we can minimize the sum of the colorfulness of the bins occuring during packing the items. We call the original problem variant $\max -B C$ and the new variant sum-BC. Note that the algorithms GreEdyFiT and OnEBin work for both variants.

Application to elevator control Before starting with the technical part of the paper, we shortly explain an interesting connection between the Online Bin Coloring problem and the relative performance of conventional elevator control and elevator control based on destination hall calls [10]. In conventional elevator control systems, a passenger specifies the desired travel direction and, later in the elevator car, her destination floor. In constrast, if the elevator system is based on destination hall calls, she specifies her destination floor already at her current floor and is immediately assigned to a serving elevator. Thus a destination hall call control system can use more information for scheduling the elevators.

An important figure for dimensioning an elevator system is the handling capacity [2]. The handling capacity is defined as the critical passenger arrival rate: If the passenger arrival is higher than the handling capacity, the system cannot cope with the resulting traffic and waiting times increase rapidly. The handling capacity is determined by considering up-peak traffic, i. e., all arriving passengers arrive at the ground floor and want to travel to the upper floors, which is the most demanding traffic situation [2].

The handling capacity is inversely related to the number of stops $S$ an elevator car does before returning to the ground floor. We now want to compare $S$ for a conventional system to that of a destination hall call system using our Bin Coloring analysis. Let us assume that the passenger arrival rate is so high that the elevators leaving the ground floor are always full (this is also assumed in the standard formula for the handling capacity [2]). Since we are only interested in the number of stops for a round trip, we can model the passenger sequence by a sequence of destinations only.

In a conventional system, passengers board the lifts in the order of their arrival at the ground floor. The stops of the resulting round trips are determined by the sub-sequences of size $P$ of the destination floor sequence. Regarding the destination floors as colors and the round trips as bins, this can be viewed as ONEBIN working on sequences with $N$ distinct colors and bin capacity $P$.

For a destination hall call system the elevator control has the possibility to reduce stops by assigning passengers with the same destination floor to the same lift and balancing the number of stops between 
the $m$ lifts. A natural strategy to do this is the GREEDYFIT algorithm, using up to $m$ bins.

As we will see in Theorem 5, the average number of colors achieved by GREEDYFIT is stochastically dominated by that of OnEBin. Applied to our elevator setting this means that the total number of stops in the destination hall call system is stochastically dominated by that of the conventional system. Since $X \leq_{\text {st }} Y$ implies $1 / X \geq_{\text {st }} 1 / Y$, we get that the handling capacity of a destination call system is higher than that of a conventional system, independent of the destination floor distribution.

\section{Markov chain models and preliminaries}

The results for both problem variants are based on the analysis of Markov chains induced by the algorithms.

\subsection{Markov chain models}

Let us start with the max-BC and consider an arbitrary Online Bin Coloring algorithm processing color sequences generated by our random model. The operation of any such algorithm can be described on a state space which encodes for every bin $i$ its current number of items $f_{i}$, the set of colors in that bin $C_{i}$ plus the maximal colorfulness attained so far. Formally, we have

$$
\begin{aligned}
& \mathcal{S}_{\max -\mathrm{BC}}:=\mathcal{S}_{\max -\mathrm{BC}}(m, B, C)=\left\{\left(f_{1}, C_{1}, \ldots, f_{m}, C_{m}, \chi\right)|0 \leq| C_{i} \mid \leq f_{i} \leq B\right. \\
&\left.\left|C_{i}\right| \leq \chi \leq \min \{B, C\}\right\} .
\end{aligned}
$$

Note that the states reachable by the operation of an algorithm may be a subset of $\mathcal{S}_{\max -\mathrm{BC}}$.

We will use $f_{i}(s), C_{i}(s)$, and $\chi(s)$ to refer to the components of state $s$. Additionally, we define $c_{i}(s):=\left|C_{i}(s)\right|$. The state $(0, \emptyset, \ldots, 0, \emptyset, 0)$ is called the initial empty state.

Suppose an Online Bin Coloring algorithm $\mathcal{A}$ is in state $s$ and receives an item of color $c$. The algorithm then changes to state $s^{\prime}$ by putting this item in one of the bins, say bin $i$. There are two cases: Either color $c$ is contained in $C_{i}(s)$, we say the color is known (in bin i), or it is not, so the color is new (in bin $i$ ). We will denote the successor state for the first case by $s^{\text {known(i) }}$ (the color $c$ is not needed to determine the successor state), for the second by $s^{\text {new }(i, c)}$. It will be convenient not to consider the new color $c$, but to deal with the random state resulting from $s$ if any new color distributed according to $\gamma$ is seen. We will use the notation $s^{\text {new }(i)}$ for this random state.

The ONEBIN algorithm is then described by the transitions

$$
s^{\prime}= \begin{cases}s^{\text {known(1) }} & \text { with probability } \gamma\left(C_{1}(s)\right), \\ s^{\text {new }(1)} & \text { with probability } 1-\gamma\left(C_{1}(s)\right),\end{cases}
$$

where we use the shortcut notation $\gamma(S):=\sum_{s \in S} \gamma(s)$. This defines a Markov chain which we denote by $\mathrm{OB}(m, B, C, \gamma)$. Note that although OneBin uses only the first bin, we consider $\mathrm{OB}(m, B, C, \gamma)$ as working on the whole state space with $m$ bins.

Similarly, we can give a Markov chain $\operatorname{GF}(m, B, C, \gamma)$ for $\operatorname{GreedyFit.~} \operatorname{GF}(s)$ is the bin GreedyFit selects for an item with a new color in state $s$. Depending on the tie-breaking rule used by the specific variant of GREEDYFit, GF $(s)$ may or may not be a random variable. We only need that $\mathrm{GF}(s)$ is one of the bins having in state $s$ the smallest number of colors.

$$
s^{\prime}= \begin{cases}s^{\operatorname{known}(i)} & \text { with probability } \gamma\left(C_{i}(s)\right) \quad 1 \leq i \leq m, \\ s^{\text {new }(\operatorname{GF}(s))} & \text { with probability } 1-\gamma\left(\bigcup_{i} C_{i}(s)\right)\end{cases}
$$

The operation of online algorithms in the sum-BC problem can be captured by a similar Markov chain model. The main difference is that the $\chi$-component is no longer the maximum of the colorfulness seen so far, but the sum. Note that the resulting Markov chains are infinite. We can use the state space

$$
\begin{aligned}
\mathcal{S}_{\text {sum-BC }}:=\mathcal{S}_{\text {sum-BC }}(m, B, C)=\left\{\left(f_{1}, C_{1}, \ldots, f_{m}, C_{m}, \chi\right) \mid\right. & 0 \leq\left|C_{i}\right| \leq f_{i} \leq B \\
& \left.\left|C_{i}\right| \leq \min \{B, C\}, \chi \in \mathbb{N}_{0}\right\} .
\end{aligned}
$$


The $\chi$-component increases each time a new color for a bin is encountered.

To avoid notational overhead, we will use the same notation for both problem variants. Therefore the sum-BC-Markov chains for OneBin and GreedyFit will be denoted by $\mathrm{OB}(m, B, C, \gamma)$ and $\mathrm{GF}(m, B, C, \gamma)$, too.

We use the notations $\operatorname{OB}(m, B, C, \gamma)_{n}$ and $\operatorname{GF}(m, B, C, \gamma)_{n}$ for the random state after $n$ steps when OneBin and GREeDyFit are started in the initial empty state. The goal of this paper is to show that, in both problem variants,

$$
\chi\left(\mathrm{GF}(m, B, C, \gamma)_{n}\right) \leq_{\mathrm{st}} \chi\left(\mathrm{OB}(m, B, C, \gamma)_{n}\right) \quad \forall n
$$

and for all parameters $m, B, C$ and color distributions $\gamma$. This kind of result is known as comparison result for Markov chains in the probability theory literature, see e.g., [19].

\subsection{A new comparison criterion}

Unfortunately, the general comparison results for Markov chains based on stochastic monotonicity [19] are not sufficient to prove stochastic dominance between GREEDYFit and OnEBin. Doisy [8] developed a comparison criterion that is not based on stochastic monotonicity, however, this result is also too weak.

Our analysis is therefore based on the following criterion, which is an extension of a result in [4]. The criterion is based on stopping times. Given a Markov chain $X=\left(X_{n}\right)_{n \in \mathbb{N}_{0}}$ on state space $\mathcal{S}$ with valuation function $\chi: \mathcal{S} \rightarrow V, V \subseteq \mathbb{N}_{0}$, we denote by $T_{X}^{v}$ the first time the Markov chain $X$ reaches a state with valuation at least $v$.

Theorem 1 Let $X=\left(X_{n}\right)_{n \in \mathbb{N}_{0}}$ and $Y=\left(Y_{n}\right)_{n \in \mathbb{N}_{0}}$ be Markov chains on state space $\mathcal{S}$ and let $\chi: \mathcal{S} \rightarrow V$ be a valuation function for some $V=\left\{0, \ldots, \chi_{\max }\right\}$. Assume that the transitions of $X$ and $Y$ are such that the value of a state is nondecreasing in each step and that $\chi\left(X_{0}\right)=\chi\left(Y_{0}\right)$. Then the following are equivalent:

1. $T_{Y}^{v} \leq_{\mathrm{st}} T_{X}^{v} \quad \forall v \in V$.

2. $\chi\left(X_{n}\right) \leq_{\text {st }} \chi\left(Y_{n}\right) \quad \forall n \in \mathbb{N}_{0}$.

Proof: Let the Markov chain $X$ be defined on the probability space $(\Omega, \mathcal{A}$, prob). The stopping time $T_{X}^{v}$ is then a random variable $T_{X}^{v}: \Omega \rightarrow \mathbb{N}_{0}$ that is defined by

$$
T_{X}^{v}(\omega):=\min \left\{n \mid \chi\left(X_{n}(\omega)\right) \geq v\right\}
$$

for each $\omega \in \Omega$. Since $\chi\left(X_{n}(\omega)\right) \geq \chi\left(X_{n^{\prime}}(\omega)\right)$ whenever $n^{\prime} \leq n$, we have the equivalence

$$
T_{X}^{v}(\omega) \leq n \Longleftrightarrow \chi\left(X_{n}(\omega)\right) \geq v
$$

which implies

$$
\operatorname{Pr}\left[T_{X}^{v} \leq n\right]=\operatorname{Pr}\left[\chi\left(X_{n}\right) \geq v\right] .
$$

Of course, analogous statements hold for $Y$ as well.

We now have the following chain of equivalences.

$$
\begin{aligned}
& \chi\left(X_{n}\right) \leq_{\text {st }} \chi\left(Y_{n}\right) \quad \forall n \in \mathbb{N}_{0} \\
\Longleftrightarrow & \operatorname{Pr}\left[\chi\left(X_{n}\right) \leq v\right] \geq \operatorname{Pr}\left[\chi\left(Y_{n}\right) \leq v\right] \quad \forall n \in \mathbb{N}_{0}, v \in V \\
\Longleftrightarrow & 1-\operatorname{Pr}\left[\chi\left(X_{n}\right) \geq v+1\right] \geq 1-\operatorname{Pr}\left[\chi\left(Y_{n}\right) \geq v+1\right] \quad \forall n \in \mathbb{N}_{0}, v \in V \\
\Longleftrightarrow & 1-\operatorname{Pr}\left[T_{X}^{v+1} \leq n\right] \geq 1-\operatorname{Pr}\left[T_{Y}^{v+1} \leq n\right] \quad \forall n \in \mathbb{N}_{0}, v \in V \\
\Longleftrightarrow & \operatorname{Pr}\left[T_{Y}^{v+1} \leq n\right] \geq \operatorname{Pr}\left[T_{X}^{v+1} \leq n\right] \quad \forall n \in \mathbb{N}_{0}, v \in V \\
\Longleftrightarrow & T_{Y}^{v+1} \leq_{\text {st }} T_{X}^{v+1} \quad \forall v \in V .
\end{aligned}
$$

Moreover, due to $\chi\left(X_{0}\right)=\chi\left(Y_{0}\right) \geq 0$ we also have $T_{Y}^{0} \leq_{\text {st }} T_{X}^{0}$.

In the sequel, we denote by $T_{X}^{v}(s)$ the stopping time for reaching a state with valuation at least $v$ when started deterministically in state in $s$. 
How can we show $T_{Y}^{v}\left(s_{0}\right) \leq_{\text {st }} T_{X}^{v}\left(s_{0}\right)$ ? In order to apply a kind of induction technique we introduce a family of Markov chains $(X(n))_{n \in \mathbb{N}}$ derived from a Markov chain $X$ as follows. The state space of $X(n)$ is $\mathcal{S} \times\{0, \ldots, n\}$ and the transitions are defined by

$$
\begin{array}{rrr}
\operatorname{Pr}\left[X(n)_{i+1}=\left(s^{\prime}, i+1\right) \mid X(n)_{i}=(s, i)\right]:=\operatorname{Pr}\left[X_{i+1}=s^{\prime} \mid X_{i}=s\right] & \forall 0 \leq i<n, \\
\operatorname{Pr}\left[X(n)_{i+1}=(s, n) \mid X(n)_{i}=(s, n)\right]:=1 & \forall i \geq n .
\end{array}
$$

The Markov chain $X(n)$ can be thought of as an time-expanded, acyclic version of the chain $X$ for the first $n$ steps. Clearly, we have

$$
\operatorname{Pr}\left[T_{X}^{v}(s)=i\right]=\operatorname{Pr}\left[T_{X(n)}^{v}((s, 0))=i\right] \quad \forall 0 \leq i<n .
$$

So in order to show $T_{Y}^{v}\left(s_{0}\right) \leq_{\mathrm{st}} T_{X}^{v}\left(s_{0}\right)$, we can prove that

$$
T_{Y(n)}^{v}\left(\left(s_{0}, 0\right)\right) \leq_{\text {st }} T_{X(n)}^{v}\left(\left(s_{0}, 0\right)\right) \quad \forall n \in \mathbb{N} .
$$

To simplify notation, we will write $T_{X(n)}^{v}(s)$ for $T_{X(n)}^{v}((s, 0))$ from now on. We have the following simple result.

Lemma 1 For any Markov chain $X=\left(X_{n}\right)_{n \in \mathbb{N}_{0}}$ on state space $\mathcal{S}$ with valuation function $\chi: \mathcal{S} \rightarrow V$, $V=\left\{0, \ldots, \chi_{\max }\right\}$, the stochastic dominance relation

$$
T_{X(n+1)}^{v}(s) \leq_{\mathrm{st}} T_{X(n)}^{v}(s)
$$

holds for all states $s, n \in \mathbb{N}_{0}$, and $v \in V$.

Proof: Consider a sample path $\omega=\left(X_{0}, X_{1}, \ldots, X_{n}\right)$ of $X(n)$ with $X_{0}=s$. Obviously, $\omega$ can be extended to a sample path $\omega^{\prime}=\left(X_{0}, X_{1}, \ldots, X_{n}, X_{n+1}\right)$ of $X(n+1)$ and all sample paths of $X(n+1)$ starting in $s$ are obtained this way. There are two cases:

1. $\chi\left(X_{n}\right) \geq v$ : For all $\omega^{\prime}$ that are extensions of $\omega$ we have $T_{X(n+1)}^{v}(s)=T_{X(n)}^{v}(s)$.

2. $\chi\left(X_{n}\right)<v$ : For those $\omega, T_{X(n)}^{v}(s)$ is infinite, whereas for any extension $\omega^{\prime} T_{X(n+1)}^{v}(s)$ is either $n+1$ or infinite, too. Thus $T_{X(n+1)}^{v}(s) \leq T_{X(n)}^{v}(s)$.

\subsection{Further preliminaries}

An important tool used frequently in this paper is the notion of mixture of random variables.

Definition 1 Let $\left(X_{m}\right)_{m \in M}$ be a family of random variables and $\Theta$ be an $M$-valued random variable. The random variable $Y$ defined by $Y:=X_{\Theta}$ (i. e., the $X$-variable to use is given by the realization of $\Theta$ ) is called a mixture and denoted by $\left[\left(X_{m}\right)_{m \in M} \mid \Theta\right]$.

An important property of $\leq_{\text {st }}$ is that it is closed under mixture, as stated in the Mixture Theorem.

Theorem 2 ([19, p. 6]) Suppose $\left[\left(X_{m}\right)_{m \in M} \mid \Theta\right]$ and $\left[\left(Y_{m}\right)_{m \in M} \mid \Theta\right]$ are two mixtures controlled by the same random variable $\Theta$ satisfying $X_{m} \leq_{\text {st }} Y_{m}$ for all $m \in M$. Then we have

$$
\left[\left(X_{m}\right)_{m \in M} \mid \Theta\right] \leq_{\mathrm{st}}\left[\left(Y_{m}\right)_{m \in M} \mid \Theta\right] .
$$

A more general version of the following theorem was proved by Strassen, see e.g., [17].

Theorem 3 (Strassen's Theorem) For two random variables $X$ and $Y$ the following are equivalent:

1. $X \leq_{\mathrm{st}} Y$

2. There is a probability space $(\Omega, \mathcal{A}, P)$ with random variables $\tilde{X}, \tilde{Y}: \Omega \rightarrow \mathbb{R}$ such that

- $\tilde{X}$ and $\tilde{Y}$ are distributed as $X$ and $Y$, respectively, and

- $\operatorname{Pr}[\tilde{X} \leq \tilde{Y}]=1$.

For two random variables $X$ and $Y$, we will frequently write $X=Y$ to mean that they have the same distribution function. 


\section{GREEDYFIT is better than ONEBIN: max-BC}

We will now apply the strategy described in Section 3.2 to the comparison of the GREEDYFiT and the ONEBIN bin coloring algorithms. The main technique is to analyze a kind of stochastic recursion for $T_{X(n)}^{v}$ based on a mixture of random variables.

Let $\mathrm{OB}=\mathrm{OB}(m, B, C, \gamma)$ for fixed parameters $m, B, C, \gamma$. In a state $s \in \mathcal{S}_{\max -\mathrm{BC}}$ OneBin does the transitions to states

$$
\begin{cases}s^{\text {known(1) }} & \text { with probability } \gamma\left(C_{1}(s)\right), \\ s^{\text {new }(1)} & \text { with probability } 1-\gamma\left(C_{1}(s)\right) .\end{cases}
$$

Using the random variable $\Theta: \mathcal{S}_{\max -\mathrm{BC}} \rightarrow \mathbb{N}$ defined by

$$
\Theta(s):= \begin{cases}1 & \text { the next color is known in bin } 1 \\ 2 & \text { the next color is new in bin } 1\end{cases}
$$

we can come up with a recursive expression for $T_{\mathrm{OB}(n)}^{v}(s)$, namely

$$
T_{\mathrm{OB}(n)}^{v}(s)= \begin{cases}0 & \chi(s) \geq v, \\ 1+\left[T_{\mathrm{OB}(n-1)}^{v}\left(s^{\text {known(1)}}\right), T_{\mathrm{OB}(n-1)}^{v}\left(s^{\text {new }(1)}\right) \mid \Theta(s)\right] & \chi(s)<v .\end{cases}
$$

This recursion and the Mixture Theorem 2 are the most important ingredients for the proofs to come.

We call two states $s, s^{\prime} \in \mathcal{S}_{\text {max-BC }}$ OB-equivalent, if the valuation, the number of items and the set of colors in bin 1 are the same in $s$ and $s^{\prime}$, i. e., if $\chi(s)=\chi\left(s^{\prime}\right), f_{1}(s)=f_{1}\left(s^{\prime}\right)$, and $C_{1}(s)=C_{1}\left(s^{\prime}\right)$. Note that ONEBIN behaves exactly the same in two OB-equivalent states and therefore the stopping times from two OB-equivalent states coincide. The following lemma gives some useful comparisons of stopping times from certain states in the $\mathrm{OB}(n)$ chains.

Lemma 2 Consider the OneBIN Markov chain $\mathrm{OB}=\mathrm{OB}(m, B, C, \gamma)$ for parameters $m, B \geq 2, C$, and color distribution $\gamma$. We have for all states $s \in \mathcal{S}_{\max -\mathrm{BC}}, n \in \mathbb{N}$, and $v \in V$ :

1. $T_{\mathrm{OB}(n)}^{v}\left(s^{\text {new }(1)}\right) \leq_{\mathrm{st}} T_{\mathrm{OB}(n)}^{v}\left(s^{\operatorname{known}(1)}\right)$, and

2. $T_{\mathrm{OB}(n)}^{v}\left(s^{\text {new }(1)}\right) \leq_{\mathrm{st}} T_{\mathrm{OB}(n)}^{v}\left(s^{\prime}\right)$ for every state $s^{\prime}$ that is OB-equivalent to $s$.

Proof: Let $s \in \mathcal{S}_{\max -\mathrm{BC}}$ be such that $\chi\left(s^{\text {new(1) }}\right)<v$ (the case $\chi\left(s^{\text {new }(1)}\right) \geq v$ is trivial).

1. Observe that both $s^{\text {new(1) }}$ and $s^{\text {known(1) }}$ have the same number of items in bin 1 , say $f$. In the case $f=0$ both states are OB-equivalent, since bin 1 is empty then. Therefore, $T_{\mathrm{OB}(n)}^{v}\left(s^{\text {new }(1)}\right)=$ $T_{\mathrm{OB}(n)}^{v}\left(s^{\text {known }(1)}\right)$.

For $f>0$ the evolution from both states will be identical after $B-f$ steps, since bin 1 is then empty again. It is therefore sufficient to show

$$
T_{\mathrm{OB}(B-f)}^{v}\left(s^{\text {new }(1)}\right) \leq_{\mathrm{st}} T_{\mathrm{OB}(B-f)}^{v}\left(s^{\text {known(1)}}\right)
$$

for all $1 \leq f<B$. We will show this by induction on $j:=B-f$.

To start the induction, consider $j=1$. There are two cases:

- $c_{1}(s)=v-2$ : We have $\operatorname{Pr}\left[T_{\mathrm{OB}(j)}^{v}\left(s^{\text {new }(1)}\right) \leq 1\right] \leq 1$ and $\operatorname{Pr}\left[T_{\mathrm{OB}(j)}^{v}\left(s^{\text {known(1)}}\right)=\infty\right]=1$.

- $c_{1}(s)<v-2$ : Then $T_{\mathrm{OB}(j)}^{v}\left(s^{\text {new }(1)}\right)=T_{\mathrm{OB}(j)}^{v}\left(s^{\text {known }(1)}\right) \equiv \infty$.

In both cases, the stochastic dominance is immediate.

Let us now consider the induction step, i. e., $j>1$. The key observation is that (since we need at least two items to close bin 1)

$$
\begin{aligned}
f_{1}\left(s^{\text {new }(1), \operatorname{known}(1)}\right) & =f_{1}\left(s^{\operatorname{known}(1), \text { new }(1)}\right) \quad \text { and } \\
C_{1}\left(s^{\text {new }(1), \operatorname{known}(1)}\right) & =C_{1}\left(s^{\text {known(1),new }(1)}\right),
\end{aligned}
$$


which means that both states are OB-equivalent. Using the Mixture Theorem 2, we can then estimate

$$
\begin{array}{lr}
T_{\mathrm{OB}(j)}^{v}\left(s^{\text {new }(1)}\right) & \\
=1+\left[T_{\mathrm{OB}(j-1)}^{v}\left(s^{\text {new }(1), \operatorname{known}(1)}\right), T_{\mathrm{OB}(j-1)}^{v}\left(s^{\text {new }(1), \mathbf{n e w}(1)}\right) \mid \Theta\left(s^{\text {new }(1)}\right)\right] & \text { by induction } \\
\leq_{\mathrm{st}} 1+T_{\mathrm{OB}(j-1)}^{v}\left(s^{\text {new }(1), \operatorname{known}(1)}\right) & \text { OB-equivalence } \\
=1+T_{\mathrm{OB}(j-1)}^{v}\left(s^{\text {known(1),new }(1)}\right) & \text { by induction } \\
\leq_{\mathrm{st}} 1+\left[T_{\mathrm{OB}(j-1)}^{v}\left(s^{\text {known }(1), \operatorname{known}(1)}\right), T_{\mathrm{OB}(j-1)}^{v}\left(s^{\text {known }(1), \mathbf{n e w}(1)}\right) \mid \Theta\left(s^{\text {known }(1)}\right)\right] & \\
=T_{\mathrm{OB}(j)}^{v}\left(s^{\operatorname{known}(1)}\right) . &
\end{array}
$$

2. Since $s$ and $s^{\prime}$ are OB-equivalent, we have

$$
\begin{aligned}
& T_{\mathrm{OB}(n)}^{v}\left(s^{\prime}\right)=T_{\mathrm{OB}(n)}^{v}(s) \\
& =1+\left[T_{\mathrm{OB}(n-1)}^{v}\left(s^{\text {new }(1)}\right), T_{\mathrm{OB}(n-1)}^{v}\left(s^{\text {known }(1)}\right) \mid \Theta(s)\right] \\
& \geq_{\mathrm{st}} 1+T_{\mathrm{OB}(n-1)}^{v}\left(s^{\text {new }(1)}\right) \\
& \geq_{\mathrm{st}} 1+T_{\mathrm{OB}(n)}^{v}\left(s^{\text {new }(1)}\right) \\
& \geq_{\mathrm{st}} T_{\mathrm{OB}(n)}^{v}\left(s^{\text {new }(1)}\right) .
\end{aligned}
$$

OB-equivalence

by 1 .

by Lemma 1

Theorem 4 Let $\mathrm{OB}$ and GF be the ONEBIN and GREEDYFiT max-BC-Markov chains for fixed parameters $m, B, C$ with $B, m \geq 2$ for some color distribution $\gamma$. We have for all states $s \in \mathcal{S}_{\max -\mathrm{BC}}, n \in \mathbb{N}$, and $v \in V$ :

$$
T_{\mathrm{OB}(n)}^{v}(s) \leq_{\mathrm{st}} T_{\mathrm{GF}(n)}^{v}(s) .
$$

Proof: The proof is by induction on $n$. Since GreedyFit is not worse than OneBin for a single step in each state $s$, we have $T_{\mathrm{OB}(1)}^{v}(s) \leq_{\mathrm{st}} T_{\mathrm{GF}(1)}^{v}(s)$.

For the induction step, suppose we know that $T_{\mathrm{OB}(n)}^{v}(s) \leq_{\mathrm{st}} T_{\mathrm{GF}(n)}^{v}(s)$ for all $s \in \mathcal{S}_{\mathrm{max}-\mathrm{BC}}$. Consider a state $s \in \mathcal{S}_{\max -\mathrm{BC}}$. Define the random variable $\Theta: \mathcal{S}_{\max -\mathrm{BC}} \rightarrow\{1, \ldots, m+1\}$ by

$$
\operatorname{Pr}[\Theta(s)=i]= \begin{cases}\gamma\left(C_{i}(s)\right) & 1 \leq i \leq m, \\ 1-\gamma\left(\bigcup_{i} C_{i}(s)\right) & i=m+1,\end{cases}
$$

i. e., $\Theta$ in a sense "selects" the GREEDYFit successor of state $s$. Using $\Theta$, we can write the recursion for the stopping time of $\mathrm{OB}$ as

$$
\begin{aligned}
& T_{\mathrm{OB}(n+1)}^{v}(s) \\
& =1+\left[T_{\mathrm{OB}(n)}^{v}\left(s^{\text {known }(1)}\right), T_{\mathrm{OB}(n)}^{v}\left(s^{\text {new }(1)}\right), \ldots, T_{\mathrm{OB}(n)}^{v}\left(s^{\text {new }(1)}\right) \mid \Theta(s)\right] .
\end{aligned}
$$

Observing that $s^{\mathrm{known}(i)}, 2 \leq i \leq m$, are OB-equivalent to $s, s^{\text {new }(\mathrm{GF}(s))}$ is either OB-equivalent to $s$ or equal to $s^{\text {new(1) }}$ we can use Lemma 2 to bound this by

$$
\leq_{\mathrm{st}} 1+\left[T_{\mathrm{OB}(n)}^{v}\left(s^{\mathrm{known}(1)}\right), \ldots, T_{\mathrm{OB}(n)}^{v}\left(s^{\text {known }(m)}\right), T_{\mathrm{OB}(n)}^{v}\left(s^{\text {new }(\mathrm{GF}(s))}\right) \mid \Theta(s)\right]
$$

which by the induction hypothesis is bounded by

$$
\begin{aligned}
& \leq_{\mathrm{st}} 1+\left[T_{\mathrm{GF}(n)}^{v}\left(s^{\text {known }(1)}\right), \ldots, T_{\mathrm{GF}(n)}^{v}\left(s^{\text {known }(m)}\right), T_{\mathrm{GF}(n)}^{v}\left(s^{\text {new }(\mathrm{GF}(s))}\right) \mid \Theta(s)\right] \\
& =T_{\mathrm{GF}(n+1)}^{v}(s) .
\end{aligned}
$$

This concludes the induction step and the proof. 
Corollary 1 Let $\mathrm{OB}$ and GF be the ONEBIN and GREEDyFiT max-BC-Markov chains for fixed parameters $m, B, C$ and color distribution $\gamma$. We have for all states $s \in \mathcal{S}_{\max -\mathrm{BC}}$, in particular the initial empty state, that

$$
\chi\left(\mathrm{GF}(s)_{n}\right) \leq_{\mathrm{st}} \chi\left(\mathrm{OB}(s)_{n}\right)
$$

for all $n \in \mathbb{N}_{0}$.

Proof: The cases $m=1$ and $B=1$ are trivial. For the remaining cases, combine Theorems 1 and 4 and the relation of $\mathrm{OB}(n)$ and $\mathrm{OB}$ as well as $\mathrm{GF}(n)$ and GF according to Equation (4).

\section{GREeDYFit is better than ONEBIN: sum-BC}

The analysis of the sum- $\mathrm{BC}$ problem is very similar to the one of max- $\mathrm{BC}$ in the preceding section. Recall that the state space of the sum-BC only differs from the one of the max- $\mathrm{BC}$ in its interpretation of the $\chi$ component: it now counts the sum of the colorfulness of all used bins instead of the maximum. Therefore, the $\chi$-component increases with every transition due to a new color. Nevertheless, recursion (5) for the stopping times is also valid for the analysis of the sum-BC.

Note that the proof of Theorem 4 is based only on Lemma 2. The proof of item 2 of Lemma 2 needs only item 1 and OB-equivalence. However, the notion of OB-equivalence introduced for the max-BC is also appropriate for the sum-BC. In particular, stopping times for two OB-equivalent states coincide also for the sum-BC-Markov chain of ONEBIN. Due to these observations, it is sufficient to prove an analogue of item 1 of Lemma 2 to establish stochastic dominance between GreEDYFiT and ONEBIN for the sum-BC. The proof uses the concept of a coupling Markov chain.

Definition 2 Let $X=\left(X_{n}\right)_{n \in \mathbb{N}_{0}}$ and $Y=\left(Y_{n}\right)_{n \in \mathbb{N}_{0}}$ be Markov chains on state space $\mathcal{S}$. A Markov chain $Z=(\tilde{X}, \tilde{Y})$ on state space $\mathcal{S} \times \mathcal{S}$ is a coupling Markov chain if $\tilde{X}$ and $\tilde{Y}$ are distributed as $X$ and $Y$, respectively. However, $\tilde{X}$ and $\tilde{Y}$ need not be independent.

Lemma 3 Consider the OneBIN Markov chain $\mathrm{OB}=\mathrm{OB}(m, B, C, \gamma)$ for parameters $m, B \geq 2, C$, and color distribution $\gamma$ for the sum-BC. We have for all states $s \in \mathcal{S}_{\text {sum-BC }}, n \in \mathbb{N}$, and $v \in V$ :

$$
T_{\mathrm{OB}(n)}^{v}\left(s^{\text {new }(1)}\right) \leq_{\mathrm{st}} T_{\mathrm{OB}(n)}^{v}\left(s^{\text {known }(1)}\right) .
$$

\section{Proof:}

We will show the stronger

$$
T_{\mathrm{OB}}^{v}\left(s^{\text {new }(1, c)}\right) \leq_{\mathrm{st}} T_{\mathrm{OB}}^{v}\left(s^{\text {known }(1)}\right)
$$

for all $c \notin C_{1}(s)$ by constructing a coupling Markov chain $Z=(X, Y)$ on a state space that is a subset of $\mathcal{S}_{\text {sum-BC }} \times \mathcal{S}_{\text {sum-BC }}$. The first component of $Z$ behaves exactly as OB started in state $s^{\text {new }(1, c)}$ and the second component as OB started in $s^{\text {known(1) }}$.

A state $\left(s^{\mathrm{n}}, s^{\mathrm{k}}\right)$ of $Z$ that can be reached from the initial state $\left(s^{\text {new }(1, c)}, s^{\text {known(1) }}\right)$ will always satisfy the invariant

- either $\chi\left(s^{\mathrm{n}}\right) \geq \chi\left(s^{\mathrm{k}}\right), f_{1}\left(s^{\mathrm{n}}\right)=f_{1}\left(s^{\mathrm{k}}\right)$, and $C_{1}\left(s^{\mathrm{n}}\right)=C_{1}\left(s^{\mathrm{k}}\right)$ or

- $\chi\left(s^{\mathrm{n}}\right)=\chi\left(s^{\mathrm{k}}\right)+1, f_{1}\left(s^{\mathrm{n}}\right)=f_{1}\left(s^{\mathrm{k}}\right)$, and $C_{1}\left(s^{\mathrm{n}}\right)=C_{1}\left(s^{\mathrm{k}}\right) \cup\{c\}$.

Since in both cases $\chi\left(s^{\mathrm{n}}\right) \geq \chi\left(s^{\mathrm{k}}\right)$, the invariant implies

$$
\operatorname{Pr}\left[T_{X}^{v}\left(s^{\text {new }(1, c)}\right) \leq T_{Y}^{v}\left(s^{\text {known(1) }}\right)\right]=1,
$$

so by Strassen's Theorem the stochastic dominance is established.

It remains to describe $Z$. The initial state is $\left(s^{\text {new }(1, c)}, s^{\text {known(1)}}\right)$, which obviously satisfies the invariant. Consider any state $\left(s^{\mathrm{n}}, s^{\mathrm{k}}\right)$ satisfying the invariant. If $s^{\mathrm{n}}$ and $s^{\mathrm{k}}$ differ at most in the $\chi^{-}$ component, then the transitions of $Z$ are such that the same happens in both components, leading to further states satisfying the invariant.

Suppose $s^{\mathrm{n}}$ and $s^{\mathrm{k}}$ differ also in the $C_{1}$-component. The transitions are then determined by the next color $c^{\prime}$ drawn according to $\gamma$ as follows:

$$
\begin{cases}\left(s^{\mathrm{n}, \text { new }\left(1, c^{\prime}\right)}, s^{\mathrm{k}, \text { new }\left(1, c^{\prime}\right)}\right) & c^{\prime} \notin C_{1}\left(s^{\mathrm{n}}\right)=C_{1}\left(s^{\mathrm{k}}\right) \cup\{c\}, \\ \left(s^{\mathrm{n}, \text { known }(1)}, s^{\mathrm{k}, \text { new }\left(1, c^{\prime}\right)}\right) & c^{\prime}=c \\ \left(s^{\mathrm{n}, \text { known }(1)}, s^{\mathrm{k}, \operatorname{known}(1)}\right) & c^{\prime} \in C_{1}\left(s^{\mathrm{k}}\right) .\end{cases}
$$


Note that all the states satisfy the invariant and that the second kind of transition leads to states which differ at most in the $\chi$-component (the other way of reaching such a state is when bin 1 is empty again). Finally, we can verify that these transitions mirror the behavior of the OB chain in each component:

$$
\begin{aligned}
& \operatorname{Pr}\left[X_{n+1}=s^{\mathrm{n}, \text { new }\left(1, c^{\prime}\right)} \mid X_{n}=s^{\mathrm{n}}\right]=1-\gamma\left(C_{1}\left(s^{\mathrm{n}}\right)\right) \text {, } \\
& \operatorname{Pr}\left[X_{n+1}=s^{\mathrm{n}, \operatorname{known}(1)} \mid X_{n}=s^{\mathrm{n}}\right]=\gamma\left(C_{1}\left(s^{\mathrm{n}}\right)\right), \\
& \operatorname{Pr}\left[Y_{n+1}=s^{\mathrm{k}, \text { new }\left(1, c^{\prime}\right)} \mid Y_{n}=s^{\mathrm{k}}\right]=1-\gamma\left(C_{1}\left(s^{\mathrm{k}}\right)\right) \text {, } \\
& \operatorname{Pr}\left[Y_{n+1}=s^{\mathrm{k}, \operatorname{known}(1)} \mid Y_{n}=s^{\mathrm{k}}\right]=\gamma\left(C_{1}\left(s^{\mathrm{k}}\right)\right) \text {. }
\end{aligned}
$$

Remark 1 The above coupling argument can be generalized for any algorithm whose decisions do not depend on $\chi(s)$, both for sum-BC and max-BC.

Theorem 5 Let $\mathrm{OB}$ and GF be the ONEBIN and GREEDYFiT sum-BC-Markov chains for fixed parameters $m, B, C$ and color distribution $\gamma$. We have for all states $s \in \mathcal{S}_{\text {sum-BC }}$, in particular the initial empty state, that

for all $n \in \mathbb{N}_{0}$.

$$
\chi\left(\mathrm{GF}(s)_{n}\right) \leq_{\text {st }} \chi\left(\mathrm{OB}(s)_{n}\right)
$$

\section{Concluding Remarks}

We introduced a new approach for the probabilistic analysis of online algorithms which is based on the concept of stochastic dominance. We applied this approach to the analysis of online algorithms for Bin Coloring problems. This analysis explains simulation results much better than the competitive analysis results existing so far and thus resolves an open problem posed in [15].

For the future it is interesting to see whether the method can be extended to analyze further Bin Coloring algorithms or more complicated probabilistic models, e.g., ones where the color sequence is generated by a Markov chain. As an example, consider the algorithm FixedColors, which assigns the items to bins based on their colors and a fixed color-to-bin assignment. In simulations we observed that this algorithm is "in-between" ONEBIN and GREEDYFiT. We also observed that GREEDYFiT outperforms ONEBIN when operating on uniform color sequences, where ONEBIN has to cope with fewer colors than GreEdyFit.

Similar techniques might also apply for other combinatorial online problems like bin packing or paging.

\section{References}

[1] S. Albers and M. Mitzenmacher. Average-case analyses of first fit and random fit bin packing. Random Structures Algorithms, 16(3):240-259, 2000.

[2] G. C. Barney. Elevator Traffic Handbook: Theory and Practice. Taylor and Francis, 2002.

[3] L. Becchetti, S. Leonardi, A. Marchetti-Spaccamela, G. Schäfer, and T. Vredeveld. Average case and smoothed competitive analysis for the multi-level feedback algorithm. Math. Oper. Res., 31(1):85$108,2006$.

[4] M. ben Mamoun, A. Bušić, J.-M. Fourneau, and N. Pekergin. Increasing convex monotone Markov chains: Theory, algorithms, and applications. In A. N. Langville and W. J. Stewart, editors, MAM 2006: Markov Anniversary Meeting, pages 189-210. Boson Books, 2006.

[5] A. Borodin and R. El-Yaniv. Online Computation and Competitive Analysis. Cambridge University Press, 1998.

[6] J. Boyan and M. Mitzenmacher. Improved results for route planning in stochastic transportation networks. In Proceedings of the 12th Annual ACM-SIAM Symposium on Discrete Algorithms, pages 895-902, 2001. 
[7] W. E. de Paepe. Complexity results and competitive analysis for vehicle routing problems. Technische Universiteit Eindhoven, 2002. Ph. D. Thesis.

[8] M. Doisy. A coupling technique for stochastic comparison of functions of Markov processes. Journal of Applied Mathematics \& Decision Sciences, 4(1):39-64, 2000. Available at http://www. hindawi. com/GetRegularIssueArticles . aspx?journal=JAMDS\&volume=4.

[9] J. E. G. Coffman, D. S. Johnson, P. W. Shor, and R. R. Weber. Markov Chains, computer proofs, and average-case analysis of best fit bin packing. In Proc. 25th Ann. ACM Symp. on Theory of Computing, pages 412-421, 1993.

[10] B. Hiller and A. Tuchscherer. Real-time destination-call elevator group control on embedded microcontrollers. In Operations Research Proceedings 200\%. Springer, 2008.

[11] B. Kalyanasundaram and K. Pruhs. Speed is as powerful as clairvoyance. J. ACM, 47(4):617-643, 2000.

[12] A. R. Karlin, S. J. Phillips, and P. Raghavan. Markov paging. SIAM J. Comput., 30(2):906-922, 2000.

[13] C. Kenyon, Y. Rabani, and A. Sinclair. Biased random walks, Lyapunov functions, and stochastic analysis of best fit bin packing. J. Algorithms, 27(2):218-235, 1998.

[14] E. Koutsoupias and C. Papadimitriou. Beyond competitive analysis. In Proceedings of the 35th Annual IEEE Symposium on Foundations of Computer Science, pages 394-400, 1994.

[15] S. O. Krumke, W. E. de Paepe, L. Stougie, and J. Rambau. Online bin coloring. In F. M. auf der Heide, editor, Proceedings of the 9th Annual European Symposium on Algorithms, volume 2161 of Lecture Notes in Computer Science, pages 74-84, 2001.

[16] M. Lin, Z. Lin, and J. Xu. Almost optimal solutions for bin coloring problems. In Proceedings of the 16th International Symposium on Algorithms and Computation, volume 3827 of Lecture Notes in Computer Science, pages 82-91. Springer, 2005.

[17] T. Lindvall. Lectures on the coupling method. Wiley Series in Probability and Mathematical Statistics. John Wiley \& Sons, 1992.

[18] M. Mitzenmacher. Bounds on the greedy routing algorithm for array networks. J. Comput. System Sci., 53:317-327, 1996.

[19] A. Müller and D. Stoyan. Comparison Models for Stochastic Models and Risks. John Wiley \& Sons, 2002.

[20] N. Naaman and R. Rom. Average case analysis of bounded space bin packing algorithms. Algorithmica, 50:72-97, 2008.

[21] G. Schaefer and N. Sivadasan. Topology matters: Smoothed competitiveness of metrical task systems. Theoret. Comput. Sci., 341(1-3):216-246, 2005.

[22] M. Scharbrodt, T. Schickinger, and A. Steger. A new average case analysis for completion time scheduling. In Proceedings of the 34th ACM Symposium on Theory of Computing, Montréal, QB, pages $170-178,2002$.

[23] H. Shachnai and T. Tamir. On two class-constrained versions of the multiple knapsack problem. Algorithmica, 29(3):442-467, 2001.

[24] H. Shachnai and T. Tamir. Polynomial time approximation schemes for class-constrained packing problems. Journal of Scheduling, 4:313-338, 2001.

[25] M. Shaked and J. G. Shanthikumar. Stochastic orders and their applications. Probability and mathematical statistics. Academic Press, 1994.

[26] A. Sinclair. Algorithms for Random Generation and Counting: A Markov Chain Approach. Progress in Theoretical Computer Science. Birkhäuser, 1993.

[27] D. D. Sleator and R. E. Tarjan. Amortized efficiency of list update and paging rules. Comm. ACM, 28(2):202-208, 1985. 